\title{
OUVRAGES REÇUS
}

L'Absolutisme éclairé. Vol. publ. par B. KÖPECZI, A. SOBOUl, É. H. BALÁzS, D. Kosáry. Budapest/ Paris, Akadémiai Kiadó/C.N.R.S., 1985, $17 \times 25$, 364 p., bibliogr. ( Colloques de Mátrafüred ").

L'Âge d'or du mécénat : 1598-1661. Colloque international, Paris, mars 1983, par Roland MoUSNIER et Jean MESNARD. Paris, C.N.R.S., 1985. $16 \times$ 24, 440 p.

Albert Camus. Textes réunis par Paul-F. SMETS, à l'occasion du $25^{\mathrm{e}}$ anniversaire de la mort de l'écrivain. Bruxelles, Émile Bruylant/Ed. de l'Université de Bruxelles, 1985. $15 \times$ 22,5, $159 \mathrm{p}$.

Michel АмIOт, Contre l'État, les sociologues : éléments pour une histoire de la sociologie urbaine en France 11900 1980). Paris, Ecole des hautes études en sciences sociales, 1986. $15 \times 23$, 304 p. (" Recherches d'histoire et de sciences sociales ", 19).

Annales de la Faculté des lettres et sciences humaines : philosophie, littérature, langues, sciences humaines, Université de Dakar, 14, 1984.

A. H. Armstrong, L'Architecture de l'univers intelligible dans la philosophie de Plotin. Trad. de l'anglais par Josiane AyouB et Danièle LETOCHA. Ottawa, Ed. de l'Université d'Ottawa, 1984. $15,3 \times 22,8,136$ p. (« Philosophica $")$.

Atti del convegno : Segno simbolo sintomo communicazione implicanze e convergenze fra filosofia, psichiatria, psicoanalisi, Chiavari, 5-6-7 dic. 1984. A cura di Adriana Dentone e Michele SchIAvone. Genève, Esagraph, 1985. $17 \times 24,328 \mathrm{p}$.

AVICENNE, Le Livre de science. I. Logique, métaphysique; II. Science naturelle, mathématiques. Trad. par Mohammad ACHENA et Henri MASSE, 2 éd. revue et corrigée par Mohammad ACHENA. Paris, "Les Belles Lettres "/ UNESCO, 1986. 12,5 × 19,5, $260 \mathrm{p}$. ("Collection UNESCO d'œuvres représentatives/série persane ").

Francis BACON, De la justice universelle. Trad. et notes J.-B. DE VAuZELles, introd. Angèle KREMER-MARIETTI. Paris, Klincksieck, 1985. $15 \times 21$, 129 p. (« Epistémologie »).

R. Barthelemy-Vogels, Charles Hyart, L'Iconographie russe de I'Apocalypse. La « mise à jour » des livres saints d'après le manuscrit $n^{\circ} 6$ de la collection Wittert appartenant d̀ la Bibliothèque générale de l'Université de Liège. Paris, "Les Belles Lettres ", 1985. 16,5 × 25,5, 214 p. + planches (« Bibliothèque de la Faculté de philosophie et lettres de l'Université de Liège ", fasc. CCXLI).

C. Bedel, R. HahN, Y. LaIssus, J. Torlais, La Curiosité scientifique au XVIII siecle: cabinets et observatoires. Paris, Hermann, 1986. $14 \times$ 21, 112 p. (" Histoire de la pensée »).

Charles Bedel, Pierre HuARd, Médecine et pharmacie au XVIII siècle. Paris, Hermann, 1986 . $14 \times 21,100$ p. (« Histoire de la pensée ").

Henti Bergson, La Pensée et le mouvant. 
Paris, P.U.F., 1985. $12,5 \times 19$, 296 p. («Quadrige »).

George Berkeley, Oeuvres. T. 1. Ed. Geneviève BrykmaN. Paris, P.U.F., 1985. $15 \times 22,432$ p. (« Épiméthée ").

François Billacois, Le Duel dans la société française des $X V^{e}-X V I I^{e}$ siècles. Essai de psychosociologie historique. Paris, E.H.E.S.S., 1986. $16 \times 24$, 540 p., bibliogr., index.

Olivier BLOCH, Le Matérialisme. Paris, P.U.F., 1985. 11,3 3 17,6, 127 p. (“ Que sais-je ?", $n^{\circ} 2256$ ).

Margarita Boladeras Cucurella, Razón critica y sociedad. De Max Weber a la escuela de Frankfurt. Barcelona, Promociones Publicaciones Universitarias, $1985.13,5 \times 20,4,345 \mathrm{p}$. (“ Biblioteca universitaria de filosofia $"$, 9).

Luc DE Brabandere, Les Infoducs. Un nouveau mot, un nouveau monde. L'informatique au macroscope. Essai. Avec la collab. de Anne MikolajcZAK. Paris/Grembloux, Duculot, 1985. $16 \times 24,149$ p. (« Perspectives $\gg)$.

Jean BRUN, L'Homme et le langage. Paris, P.U.F., 1985. 13,5 $\times 21,5$, $258 \mathrm{p}$.

Jean Chateau, L'Humanisation ou les premiers pas des valeurs humaines. Bruxelles, Pierre Mardaga, 1985. 15 $\times 22,257$ p. (« Psychologie et sciences humaines $")$.

Jacques Chonchol, Guy Martiniére, L'Amérique latine et le latinoaméricanisme en France. Préf. Antoine BlanCA. Paris, L'Harmattan, 1985. $16 \times 24,332$ p., index, annexe.

Jeannette Colombel, Jean-Paul Sartre. 2/ Une cuvre aux mille têtes. Textes et débats. Paris, L.G.F., 1986. $11 \times$ 16,5, 762 p., bibliogr. (« Le Livre de poche. Biblio essais ", 5013).

Jean-Pierre COMETTI, Robert Musil ou l'allernative romanesque. Paris, P.U.F., 1985. $15 \times 21,7,286$ p. ("Perspectives critiques").

La Communication. Association de psychologie scientifique de langue francaise, éd., Symposium, Montpellier, 1983, sous la dir. de NOIZET, BELANGER, Bresson. Paris, P.U.F., 1985. 14 $\times \quad 22, \quad 352$ p. ("Psychologie d'aujourd'hui ").

La Correspondance d'Erasme et l'épistolographie humaniste. Colloque international tenu en nov. 1983. Bruxelles, Éd. de l'Université de Bruxelles, 1985. $16 \times 24,237$ p., index ( $~$ Travaux de l'Institut interuniversitaire pour l'étude de la Renaissance et de l'Humanisme ", VII).

Pierte Costabel, L'Enseignement classique au XvIJ⿸ siècle : collèges et universilés. Paris, Hermann, 1986. $14 \times$ 21,168 p. (" Histoire de la pensée ").

Monique CotTRET, La Bastille d̀ prendre: histoire et mythe de la forteresse royale. Préf. Pierre Chaunu. Paris, P.U.F., 1986. $15 \times 18,208$ p. (« Histoires $\gg)$.

Michèle Crampe-Casnabet, Condorcet, lecteur des Lumieres. Paris, P.U.F., 1985. $11,5 \times 17,5,128$ p. (« Philosophies ").

Elisabeth Crawford, The Beginnings of the Nobel Institution. The Science Prizes, 1901-1915. New York/Paris, Cambridge University Press, 1984. 16 $\times 23,5,281$ p., bibliogr., index.

Culture et idéologie après le Concile de Trente: permanences et changements. Études réunies par Michel Plaisance. Abbeville, Imprimerie F. Paillart, 1985. 15,5 × 24, 221 p. (" Université de Paris VIIl, Presses universitaires de Vincennes. Documents et travaux de l'équipe de recherche Culture et Société au XVI ${ }^{e}$ siècle $"$, t. V).

Philip J. Davis, Reuben Hersch, L'Univers mathématique. Traduit et adapté par L. Chambadal. Paris, GauthierVillars, 1985. 17,5 × 26,3, 416 p., ill.

Christian DELMAS, Mythologie et mythe dans le theâtre français: 1650-1676. Genève, Droz, 1985. $16 \times 24,400$ p. (" Histoire des idées et critique littéraire "), 236).

Philippe DENIS, Les Églises d'étrangers en pays rhénans (1538-1564). Paris, "Les Belles Lettres ", 1984. 16,5 x 24,5, 696 p., index (« Bibliothèque de la Faculté de philosophie et lettres de l'Université de Liège", fasc. CCXLII).

Dictionnaire des néologismes officiels. Tous les mots nouveaux. Avec en annexe l'ensemble des textes legislatifs 
et réglementaires sur la langue française. Ouvrage réalisé sous la dir. d'Alain FANTAPIE et Marcel BrulE. Paris, Franterm (diff. Nathan), 1984. $14 \times 21,5,544 \mathrm{p}$.

Béatrice DIDIER, La Musique des Lumières. Diderot, L' " Encyclopédie ", Rousseau. Paris, P.U.F., 1985. 13,5 $\times 21,5,480$ p. ("Ecriture »).

Manuel de Dieguez, Jésus. Paris, Fayard, 1985. 15,5 × 23,5, $496 \mathrm{p}$.

Différences, valeurs, hiérarchie. Textes offerts à Louis Dumont, sous la dir. de Jean-Claude Galey. Paris, E.H.E.S.S., 1984. $16 \times 24,520$ p.

Hervé DUMEZ, L'Économiste, la science et le pouvoir. Le cas Walras. Paris, P.U.F., 1985. $15 \times 21,5,272$ p. ("Sociologies ").

Brian EALSEA, Science et philosophie. Une révolution, 1450-1750. La chasse aux sorcières. Descartes. Copernic. Kepler. Trad. de l'anglais par Nina GODNEFF. Paris, Ramsay, 1986. $15 \times$ 25, $336 \mathrm{p}$.

Enseignement et diffusion des sciences en France au $x$ vIIJ siècle. Sous la dir. de René TAton. Paris, Hermann, 1986. $14 \times 21,778$ p., annexes, bibliogr., index ( $«$ Histoire de la pensée »).

“ L'Épistémologie des sciences sociales : statut scientifique, valeurs et institutionnalisation ", Revue internationale des sciences sociales, 102, vol. XXXVI, $n^{\circ} 4,1984$.

D'Erasme à Campanella. Textes de Roland Crahay, éd. par Jacques MarX. Bruxelles, Ed. de l'Université de Bruxelles, 1985. $16 \times 24,161 \mathrm{p}$. (« Problèmes d'histoire du christianisme »).

« Espaço público ", Revista de comunicacao e linguagens, 2, dez. 1985.

Bernara D'ESPAGNAT, Une incertaine réalité. Le monde quantique, la connaissance et la durée. Paris, GauthierVillars, 1985. 15,5 x 24, X + 310 p., bibliogr., index.

L'Étude et la mise en valeur du patrimoine industriel. $4^{e}$ conférence internationale, Lyon-Grenoble, sept. 1981. Paris, C.N.R.S., 1985. $16 \times 24$, 496 p. ( a Colloques internationaux du C.N.R.S. »).

François FEDIER, Interprétation. Paris,
P.U.F., 1985. $15 \times 21,5,144$ p. ("Epiméthée »).

Jean FLORI, L'Essor de la chevalerie: $X I^{e}-X I I^{e}$ siècles. Préf. de Léopold Genicot. Genève, Droz, 1986. $16 \times$ 24, 416 p. (" Travaux d'histoire éthico-politique "), 46).

Guillermo Folch Jou, María Pilar MiLLÁN GuITARTE, La Farmacia en Zaragoza a traves del tiempo. Madrid, Ed. por el Colegio de Farmacéuticos de Zaragoza en colaboración con el Departamento de Historia de la Farmacia y Legislacion farmacéutica de la Universidad Complutense de Madrid, 1985. $19 \times 25,5,218$ p., reprod.

Eudaldo Forment Giralt, Fenomenologia descriptiva del Lenguaje. Segunda edición. Barcelona, Promiciones Publicaciones Universitarias, 1984. 12,5 × 19, $352 \mathrm{p}$.

Eudaldo FORMENT, Introduccion a la metafisica. Barcelona, Ed. Universitat de Barcelona, 1984. 16,7 $\times 23,7$, 202 p., bibliogr.

La France et l'Italie du temps de Mazarin. Sous la dir. de Jean SERRoY. Grenoble, Presses Universitaires de Grenoble, $1986.16 \times 24,350$ p. (« Actes de colloque $»)$.

Jacques Gadille, Régis Ladous, Des sciences de la nature aux sciences de l'homme. Paris/Lyon, Vrin/Institut interdisciplinaire d'études épistémologiques, 1984: 16,5 $\times$ 24,5, $295 \mathrm{p}$. ("Science, histoire, philosophie »).

Jean-Pierre Goubert, La Conquête de l'eau. Introd. d'Emmanuel LE ROY LAdurie. Paris, Laffont, 1985. $16 \times$ 24, 304 p., pl. (" Les Hommes et l'histoire $\gg)$.

Henri GouHIER, Blaise Pascal. Conversion et apologétique. Paris, Vrin, 1986. 16 $\times 24,268$ p. ( " Bibliothèque d'histoire de la philosophie $")$.

Arsenij GoulyGA, Emmanuel Kant, une vie. Trad. du russe Jean-Marie VAYSSE, suivi de En quel sens sommes-nous tous kantiens? par Jean-Marie Vaysse. Paris, Aubier Montaigne, 1985. $14 \times 22,352 \mathrm{p}$.

Graeco-latina mediolanensia. Istituto di Filologia Classica. Milano, CisalpinoGoliardica, 1985. $17 \times 24,206$ p. ( ' Universita' degli studi di Milano, Facoltà di lettere e filosofia, Quaderni di ACME $", 5$ ). 
Joseph GRUNFELD, Changing Rational Standards. A Survey of Modern Philosophy of Science. Lanham/New York/London, University Press of America, 1985. 13,6 × 20,2, 171 p., index.

G. Th. Guilbaud, Leçons d'd peu près. Paris, Christian Bourgois, 1985. 13,2 $\times 20,235$ p., index.

Jürgen Habermas, Après Marx. Préf. de Jean-René LADMIRAL, trad. de l'allemand J.-R. LADMIRAL, Marc BUHOT DE Launay. Paris, Fayard, 1985. 14 $\times 22,348$ p. (《 Espace politique »).

Roger HahN, René TATON, Ecoles techniques et militaires au xvilie siècle. Paris, Hermann, 1986. $14 \times 21$, 288 p. (" Histoire de la pensée ").

L'Homme et l'espace dans la littérature, les arts et l'histoire en Espagne et en Amérique latine au $x I X^{e}$ siècle. Université de Lille III, éd. Claude Dumas. Villeneuve-d'Ascq, Presses Universitaires de Lille, $1985.16 \times 23$, 316 p. ("Travaux et recherches").

L'Homme et ses normes. $2^{\epsilon}$ partie. Nantes, Presses de l'Université de Nantes, 1985. 16,5 × 23, 95 p. (« Textes et langages », XI).

Daisâku IKEDA, La Vie à la lumière du Bouddhisme. Trad. de l'anglais par Paul Couturiau. Monaco, Ed. du Rocher, 1985. 14,5 × 23,2, $328 \mathrm{p}$.

L'Imitation : alienation ou source de liberté ? Ecole du Louvre, Paris, sept. 1984. Paris, La Documentation francaise, $1985.16 \times 24,408$ p. (") Rencontres de l'Ecole du Louvre ").

Wolfgang ISER, L'Acte de lecture : théorie de l'effet esthétique. Trad. de l'allemand Evelyne SZNYCER. Bruxelles, Mardaga, 1985. $15 \times 22,398 \mathrm{p}$. ("Philosophie et langage").

Jules Ferry, fondateur de la République. Actes du colloque organisé par l'E.H.E.S.S. présentés par François Furet. Paris, E.H.E.S.S., 1985. $16 \times$ 24, 256 p. (" Civilisations et sociétés ", 72).

Joseph Juszezak, Éloge de la métaphysique. Paris, S.E.D.E.S., 1985. $11 \times$ $17,5,145 \mathrm{p}$.

Joseph Juszezak, Les Sources du symbolisme. Paris, S.E.D.E.S., 1985. $11 \times$ $17,5,143 \mathrm{p}$.

Georges Kalinowski, Sémiotique et phi- losophie : à partir et à l'encontre de Husserl et de Carnap. ParisAmsterdam/Malakoff, Hadès-Benjamins/Distique, $1985.14 \times 21,290 \mathrm{p}$. (" Actes sémiotiques »).

Angèle KrEMER-MARIETtI, Michel Foucault. Archéologie et généalogie. Réed. Paris, L.G.F., 1985. $11 \times 16,5$, 286 p. ("Le Livre de poche. Biblio essais ", 4036).

Régis LaDOUs, Darwin, Marx, Engels, Lyssenko et les autres. Paris/Lyon, Vrin/Institut interdisciplinaire d'études épistémologiques, 1984. 16,5 X $24,5,148 \mathrm{p}$., index (" Science, histoire, philosophie ").

Yves Laissus, Jean ToRLaIS, Le Jardin du Roi et le Collège royal dans l'enseignement des sciences au $x$ III $^{e}$ siècle. Paris, Hermann, 1986. $14 \times 21,96$ p. ("Histoire de la pensée ").

Silvia LEMPEN-RicCl, Le Sens de l'imagination. Étude comparative sur la structure de l'image et l'acte d'imaginer comme pouvoir de la conscience. Genève, Georg \& Cie, 1985. $16 \times 23$, $292 \mathrm{p}$.

Jules LEQUIER, Comment trouver. Comment chercher une première vérité. Paris, Ed. de l'Éclat, 1985. 12,5 × 19, 128 p. (" Philosophie imaginaire »).

Bernard LeWIS, Le Retour de l'islam. Trad. de l'anglais Tina Jolas, Denise Paulme. Paris, Gallimard, 1985. 14 $\times 23,432$ p. (" Bibliothèque des histoires 1$)$.

La Linguistique fantastique. Sous la dir. de Sylvain Auroux, Jean-Claude Chevalier, Nicole JacquesChaquin, Christiane MarchelloNızıA. Paris, Denoël/J. Clims, 1985. $15 \times 23,380 \mathrm{p}$.

Francisco LóPEZ Frias, Ética y política. En torno al pensamiento de J. Ortega y Gasset. Segunda edición, Prólogo de Julian Marias. Barcelona, Promociones Publicaciones Universitárias, 1985. $14 \times 20,5,375$ p (« Biblioteca universitaria de filosofia $", 4)$.

Louis Vallon ou la politique en liberté. De Jaurès d̀ de Gaulle. Textes réunis et présentés par Gérard Brun. Paris, Economica, 1986. 15,5 × 24, 280 p., index.

Manifeste du Cercle de Vienne et autres écrits. Carnap, Hahn, Neurath, Schlick, Waismann, Wittgenstein. 
Sous la dir. d'Antonia Soulez, textes trad. de l'allemand par B. CASSIN, C. Chauvire, A. Guitard, J. Sebestik, A. Soulez, J. Vickers. Paris, P.U.F., 1985. 13,5 × 21,5, 364 p. ("Philosophie d'aujourd'hui ").

Les Manuscrits mathématiques de Marx. Etude et présentation par Alain AlCoufFe, première traduction française. Paris, Economica, 1985. 15,5 $\times$ 24,351 p., index.

Marx en perspective. Actes du colloque organisé par l'École des hautes études en sciences sociales, Paris, déc. 1983, textes réunis par Bernard CHAvaNCE. Paris, E.H.E.S.S., 1985. $15 \times 22,5$, $721 \mathrm{p}$.

Lloyd DE MAuse, Les Fondations de la psychohistoire. Trad. de l'américain Sean WILDER, Jean-Maurice BIZIERE. Paris, P.U.F., 1986. $15 \times 22,296$ p (" Perspectives critiques ").

Solange MERCIER-JosA, Retour sur le jeune Marx. Deux études sur le rapport de Marx à Hegel. Paris, Méridiens Klincksieck, 1986. $16 \times 23$, 195 p. (" Philosophie »).

Marco MESSERI, Causa e spiegazione. La Fisica di Pierre Gassendi. Milano, Franco Angeli, 1985. $14 \times 22,170 \mathrm{p}$. ("Collana di filosofia », 230-18).

Chantal Millon-Delsol, Essai sur le pouvoir occidental. Paris, P.U.F., 1985. $15 \times 21,5,256$ p. (《Questions »).

"Mircea Eliade ", Cahier de l'Herne. Dir. Constantin Tatou. Paris, L.G.F., 1985. $11 \times 17,415$ p. (« Le Livre de poche. Biblio essais $"$, 4033).

Misère de la sociobiologie. Ed. Patrick TORT. Paris, P.U.F., 1985. $15 \times 22$, $192 \mathrm{p}$.

Wolfgang J. Mommsen, Max Weber et la politique allemande: 1890-1920. Trad. de l'allemand. Paris, P.U.F., 1985. $15 \times 22$, 552 p. ("Sociologies $")$.

Jean-Pierre MOREL, Le Roman insupportable. L'Internationale littéraire et la France (1920-1932). Paris, Gallimard/NRF, 1985. $14 \times 22,5,488$ p. ( Bibliothèque des idées ").

Robert J. MORRISSEY, La Rêverie jusqu'à Rousseau. Recherches sur un topos littéraire. Paris, Klincksieck, 1984. $15 \times$ 23, 184 p. (« French Forum Monographs $», 55)$.

Serge Moscovıcı, L'Âge des foules : un traité historique de psychologie des masses. Nouvelle édition entièrement refondue. Bruxelles, Complexe, 1985. $12 \times 18,512$ p. (《 Historiques », 23).

Mouvements populaires et conscience sociale : $X V I-X I X^{e}$ siècles. Actes du colloque de Paris, 24-26 mai 1984, recueillis et présentés par Jean NicoLAS. Paris, Maloine, 1985. $17 \times 25$, 773 p. (“ C.N.R.S.-Université Paris VII )).

Robert MusIL, Pour une évaluation des doctrines de Mach. Ed. critique établie par Paul-Laurent Assoun avec une étude-préface Musil, lecteur de Mach et une étude-postface De Mach à la philosophie-sans-qualités. Paris, P.U.F., 1985. 13,5 × 20,5, 222 p. ("Philosophie d'aujourd'hui »).

Mythes et représentations du temps. Recueil préparé par Dorian TIFFENEAU. Paris, C.N.R.S./Centre régional de publication de Paris, 1985. 15,5 $\times 24,162$ p. (« Phénoménologie et herméneutique $")$.

Naissance de l'ethnologie? Anthropologie et missions en Amérique, $\mathrm{XVI}^{\mathrm{e}} \mathrm{xVIII^{e }}$ siècle. Textes rassemblés et présentés par Claude Blanckaert. Paris, Cerf, 1985. 13,5 × 21,5, 267 p. ( S Sciences humaines et religion $")$.

Jean Negron,, Le Savoir-vivre intellecluel: de Durkheim à Bernard-Henry Lévy. Paris, Orban, 1985. $14 \times 22$, $252 \mathrm{p}$.

Isaac Newton, De la gravitation ou les fondements de la mécanique classique. Introduction, traduction et notes de Marie-Françoise Biarnals. Paris, « Les Belles Lettres », 1985. $16 \times 24$, 192 p., index, bibliogr. (« Science et humanisme $"$ ).

Objektivationen des Geistigen. Beiträge zur Kulturphilosophie. Wolfdietrich SCHMIED-Kowarzik, Hrsg. Berlin, Dietrich Reimer Verlag, 1985. 13,5 $\times$ $20,7,426$ p. ( ( Schriften zur Kultursoziologie ", Bd 5).

Ruprecht PAQuE, Le Statut parisien des nominalistes. Recherches sur la formation du concept de réalité de la science moderne de la nature. Guillaume d'Occam, Jean Buridam et Pierre d'Espagne, Nicolas d'Autrecourt et Grégoire de Rimini. Trad. de l'allemand par Emmanuel MARTI- 
NEAU. Paris, P.U.F., 1985. $15 \times 21,5$, 413 p. ("Épiméthée ").

Jeanne Parain-Vial, Philosophie des sciences de la nature : tendances nouvelles. Préf. de René Poirier. Paris, Klincksieck, 1985. $15 \times 21,272$ p. ( Épistémologie »).

Jean-Claude PARIEnTE, L'Analyse du langage à Port-Royal : six études logicogrammaticales. Paris, Minuit, 1985. $14 \times 22,416$ p. ( 4 Le Sens commun $\gg)$.

Bernard Paulre, La Causalité en économie. Signification et portée de la modélisation structurelle. Lyon, Presses Universitaires de Lyon, 1985. $14 \times$ 20,5, 440 p. ("Science des systèmes ").

Jean PhILEIDE, L'Harmonie des paradoxes. Paris, La Pensée universelle, 1985. $13,8 \times 18,160 \mathrm{p}$.

" Philosophie et littérature », Annales de l'Institut de philosophie et de sciences morales, 1985.

Georges Poulet, La Pensée indéterminée. I. De la Renaissance au romantisme. Paris, P.U.F., 1985. 13,5 X 21,5, 304 p. ("Ecriture ").

Prace naukowe $A E, 308,1985$.

" Problèmes et méthodes de la biographie. Actes du colloque, Sorbonne 3-4 mai 1985 ", Sources. Travaux historiques, 3-4, 1985.

Pietro ReDond, Galilée hérétique. Trad. de l'italien par Monique AYMARD. Paris, Gallimard, 1985. $14 \times 22,5$, $450 \mathrm{p}$. ("Bibliothèque des histoires ").

Julien RIES, Les Chemins du sacré dans l'histoire. Paris, Aubier Montaigne, 1985. 13,5 × 22, 277 p. (" Présence et pensée $)$ ).

Marc Sautet, Patrick Boussignac, Nietzsche pour débutants. Paris, La Découverte, 1986. $14 \times 21,196 \mathrm{p}$.

Gershom SCHOLEM, La Mystique juive : les themes fondamentaux. Trad. Maurice Hayoun, Paris, Cerf, 1985. $15 \times 24,280$ p. (" Patrimoines) Judaïsme ").

« Sciences et anthropologie. Actes du colloque Colins de Ham ", Philosophie, XI, 1985.

Sciences \& Avenir, 458, avril 1985 ; 459, mai $1985 ; 463$, sept. $1985 ; 464$, oct. 1985.

Sciences et symboles. Les voies de la connaissance. Colloque de Tsukuba, prés. par Michel Cazenave. Paris, Albin Michel/France-Culture, 1986. $14,5 \times 22,5,453 \mathrm{p}$.

Les Scientifiques et leurs alliés. Textes choisis par Michel Callon et Bruno LATOUR, trad, par Dominique EBNÖTHER. Paris, Pandore, 1985. 14,5 X 20,5, 255 p., bibliogr. (" Collection Pandore ", $\left.n^{\circ} 4\right)$.

Septentrion. Revue de culture néerlandaise. $14^{e}$ année, 2, 1985.

Paul-F. SMETS, Albert Camus, dans le premier silence... et au-delà. Suivi de Albert Camus, chroniqueur judiciaire d̀ "Alger-Républicain 》 en 1939. Bruxelles, J. Goemaere, 1985. 11,3 $\times$ 18, 212 p., bibliogr.

André Stanguennec, Hegel critique de Kant. Paris, P.U.F., 1985. 13,5 × 21,5, 356 p. (" Philosophie d'aujourd'hui ").

Maurice TORRELL, Le Droit international humanitaire. Paris, P.U.F., 1985. $11,3 \times 17,2,128$ p. (« Que saisje ? $», 2211)$.

Louis VAx, La Poésie philosophique. Paris, P.U.F., 1985. 13,5 × 21, 200 p.

«Vienne : 1880-1938 ", Revue d'esthetique, 9, 1985.

Simone Weil, Philosophie, Religion, Politik. Herausgegeben von Heinz Robert SCHLETTE und André DevauX. Frankfurt am Main, Knecht, 1985. $13,5 \times 21,320 \mathrm{p}$.

Rudolf et Margot Wittkower, Les Enfants de Saturne. Psychologie et com. portement des artistes de l'Antiquité à la Révolution française. Paris, Macula, 1985. $16 \times 24,413$ p., bibliogr., index, ill.

Francis WolfF, Socrate. Paris, P.U.F., 1985. $11,5 \times 17,5,128 \mathrm{p}$. (« Philosophies »).

Christoph von Wolzogen, Zur Geschichte des Dietrich Reimer Verlages, 18451985. Berlin, Dietrich Reimer Verlag, 1986. $13,5 \times 21,83 \mathrm{p}$.

María Zambrano, Les Clairières du bois. Trad. française de Marie LAFFraN. QUE. Toulouse, Association des publications de l'Université de Toulouse-Le Mirail, 1985. $18 \times 24,160 \mathrm{p}$. (" Publications de l'Université de Toulouse-Le Mirail », série A, t. 53). 